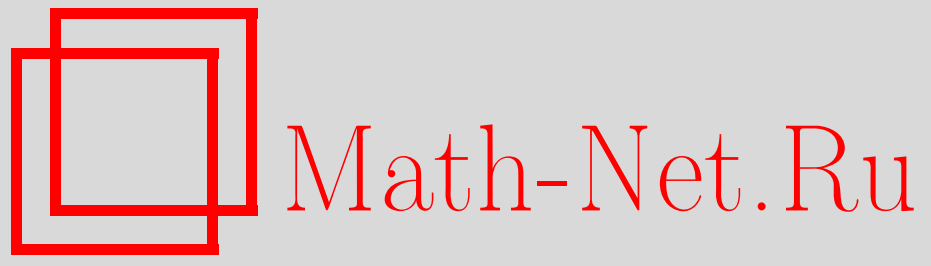

Общероссийский математический портал

М. Ю. Вагина, М. М. Кипнис, Устойчивость нулевого решения дифференциального уравнения с запаздываниями, Матем. заметки, 2003, том 74, выпуск 5, 786-789

DOI: https://doi.org/10.4213/mzm603

Использование Общероссийского математического портала Math-Net.Ru подра- 
зумевает, что вы прочитали и согласны с пользовательским соглашением http://www . mathnet.ru/rus/agreement

Параметры загрузки:

IP : 3.89 .185 .249

26 апреля 2023 г., 16:27:06

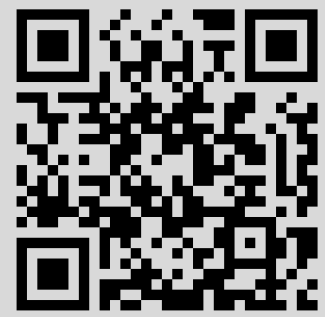




\section{УСТОЙЧИВОСТЬ НУЛЕВОГО РЕШЕНИЯ ДИФФЕРЕНЦИАЛЬНОГО УРАВНЕНИЯ С ЗАПАЗДЫВАНИЯМИ}

\section{М.Ю. Вагина, М.М. Кипнис}

Введение. Проблема устойчивости нулевого решения дифференциального уравнения

$$
\frac{d x}{d t}=-\sum_{k=1}^{n} a_{k} x\left(t-\tau_{k}\right)
$$

сводится к проблеме расположения корней характеристического уравнения

$$
p+\sum_{k=1}^{n} a_{k} e^{-p \tau_{k}}=0 .
$$

В [1] дается следующее решение этой задачи для $n=2$.

УтвеРЖ ДЕние [1, Proposition 1.2.9]. Пусть $a_{1}, a_{2}, \tau_{1}, \tau_{2} \in(0, \infty)$. Условие $a_{1} \tau_{1}+a_{2} \tau_{2}<1$ является достаточным, а условие $a_{1} \tau_{1}+a_{2} \tau_{2}<\pi / 2$ необходимым для того, чтобы все корни уравнения (2) имели отричательные вещественные части.

Мы намерены показать, что достаточное условие утверждения можно ослабить до $a_{1} \tau_{1}+a_{2} \tau_{2}$ $<\pi / 2$ (теорема 1 ), а необходимое условие ошибочно (теорема 2 ).

Работа вьполнена при финансовой поддержке Челябинского государственного педагогического университета, грант ГРГ 55/02/Ц. 
Аналогично уравнению (1) интегродифференциальное уравнение

$$
\frac{d x}{d t}=-\int_{0}^{\infty} a(\tau) x(t-\tau) d \tau
$$

где $a(\tau)>0$ при $\tau>0$. И здесь для устойчивости нулевого решения в [1] указано достаточное условие $\int_{0}^{\infty} \tau a(\tau) d \tau<1$. Мы намерены ослабить это условие до $\int_{0}^{\infty} \tau a(\tau) d \tau<\pi / 2$.

\section{1. Дифференциальное уравнение (1).}

Tеорема 1. Пусть $a_{k}, \tau_{k} \in(0, \infty), 1 \leqslant k \leqslant n$. Eсли $\sum_{k=1}^{n} a_{k} \tau_{k}<\pi / 2$, то все корни уравнения (2) имеют отрицательные действительные части.

ДоказАтЕльство. Пусть $\varphi(p)=p+\sum_{k=1}^{n} a_{k} e^{-p \tau_{k}}$. Рассмотрим движение переменной $p$ по контуру $K_{R}$ комплексной плоскости, содержащему отрезок $I_{R}: p=-i \omega,-R \leqslant \omega \leqslant R$, и полуокружность $C_{R}: p=R e^{-i \omega},-\pi / 2 \leqslant \omega \leqslant \pi / 2$. По принципу аргумента для доказательства теоремы достаточно показать, что $\Delta \operatorname{Arg} \varphi(p)=0$ при движении $p$ по $K_{R}$ при достаточно болшших $R$. Это будет означать отсутствие корней $\varphi(p)$ в правой полуплоскости. Очевидно, $\Delta \operatorname{Arg} \varphi(p) \rightarrow \pi$ при движении $p$ по $C_{R}$, если $R \rightarrow \infty$. Рассмотрим движение по отрезку $p=-i \omega, 0 \leqslant \omega \leqslant R$. Обозначим

$$
u(\omega)=\operatorname{Re} \varphi(-i \omega)=\sum_{k=1}^{n} a_{k} \cos \omega \tau_{k}, \quad v(\omega)=\operatorname{Im} \varphi(-i \omega)=-\omega+\sum_{k=1}^{n} a_{k} \sin \omega \tau_{k} .
$$

Для завершения доказательства нам достаточно показать, что из условий $u(\omega)=0, \omega>0$ следует $v(\omega)<0$, ибо тогда $\Delta \operatorname{Arg} \varphi(p) \rightarrow-\pi / 2$ при движении $p$ по отрезку $p=-i \omega, 0 \leqslant \omega \leqslant R, R \rightarrow \infty$ и вследствие симметрии $\Delta \operatorname{Arg} \varphi(p) \rightarrow-\pi$ на $I_{R}$, а поэтому $\Delta \operatorname{Arg} \varphi(p)=0$ на $K_{R}$.

Пусть $u(\omega)=0$. Вследствие элементарного неравенства $x+\cos x-(\pi / 2) \sin x \geqslant 0, x \geqslant 0$, получим при $\omega>0$

$$
\omega \sum_{k=1}^{n} a_{k} \tau_{k}+\sum_{k=1}^{n} a_{k} \cos \omega \tau_{k}-\left(\frac{\pi}{2}\right) \sum_{k=1}^{n} a_{k} \sin \omega \tau_{k} \geqslant 0
$$

Отсюда в силу (4), равенства $u(\omega)=0$ и условия $\sum_{k=1}^{n} a_{k} \tau_{k}<\pi / 2$ получим

$$
\left(\frac{\pi}{2}\right) \sum_{k=1}^{n} a_{k} \sin \omega \tau_{k} \leqslant \omega \sum_{k=1}^{n} a_{k} \tau_{k}<\frac{\omega \pi}{2}
$$

вследствие чего $v(\omega)<0$. Теорема 1 доказана.

Поскольку для уравнения

$$
\frac{d x}{d t}=-a x(t-\tau), \quad a, \tau \in(0, \infty)
$$

устойчивость нарушается при $a \tau>\pi / 2$ [2, гл. III, п. 3], легко показать, что постоянная $\pi / 2$ в теореме 1 неулучшаема. Для этого следует положить в (1) $n=2, a_{1}=a_{2}, \tau_{1}=\tau_{2}$. Тем не менее, следующая теорема 2 показьвает, что нулевое решение уравнения (1) может быть устойчивым при сколь угодно большой величине $\sum_{k=1}^{n} a_{k} \tau_{k}$.

ТЕОРема 2. Если $n=2, a_{1}=a_{2}, u a_{1} \tau_{1}<1 / 2$, то при любом $\tau_{2} \in(0, \infty)$ все корни уравнения (2) имеют отрицательные действительные части. 
ДокАЗАТЕльство. В обозначениях доказательства предыдущей теоремы $\left(n=2, a_{1}=a_{2}\right)$ достаточно показать, что из $u(\omega)=0, \omega>0$ следует $v(\omega)<0$. Действительно, пусть $u(\omega)=0$. Тогда $\cos \omega \tau_{2}=-\cos \omega \tau_{1}$, поэтому

$$
\left|\sin \omega \tau_{2}\right|=\sqrt{1-\cos \omega \tau_{2}} \sqrt{1+\cos \omega \tau_{2}} \leqslant \sqrt{2} \sqrt{1-\cos \omega \tau_{1}}=2\left|\sin \left(\omega \tau_{1} / 2\right)\right| .
$$

Вследствие этого

$$
\begin{aligned}
v(\omega) & =-\omega+a_{1}\left(\sin \omega \tau_{1}+\sin \omega \tau_{2}\right) \\
& \leqslant-\omega+a_{1}\left(\sin \omega \tau_{1}+2\left|\sin \left(\frac{\omega \tau_{1}}{2}\right)\right|\right) \leqslant-\omega+2 a_{1} \omega \tau_{1} .
\end{aligned}
$$

Если $a_{1} \tau_{1}<1 / 2$, то $v(\omega)<0$. Теорема 2 доказана.

2. Интегродифференциальное уравнение (3). В уравнении (3) предполагается, что ядро $a(\tau)$ положительно, непрерывно в области $\tau \geqslant 0$ и $\int_{0}^{\infty} a(\tau) d \tau$ сходится. Начальные условия $x(t)=\phi(t),-\infty<t \leqslant 0$, задаются непрерьвной, ограниченной на $(-\infty, 0)$ функцией $\phi$ и гарантируют существование единственного решения $x(t), t \in(0, \infty)$ [3, гл. I, п. 2]. Умножим обе части уравнения на функцию, равную 1 при $t \geqslant 0$ и равную 0 при $t<0$, а затем применим преобразование Лапласа к обеим частям. Получим, что устойчивость нулевого решения зависит от расположения корней характеристического уравнения

$$
p+\int_{0}^{\infty} a(\tau) e^{-p \tau} d \tau=0 .
$$

ТЕОРема 3. Если $\int_{0}^{\infty} \tau a(\tau) d \tau<\pi / 2$, то все корни уравнения (5) имеют отричательные действительные части.

ДокАЗАтЕЛЬСтво. Обозначим

$$
\varphi(p)=p+\int_{0}^{\infty} a(\tau) e^{-p \tau} d \tau
$$

и рассмотрим движение переменной $p$ по контуру $K_{R}$, описанному в доказательстве теоремы 1 . Ввиду сходимости $\int_{0}^{\infty} a(\tau) d \tau$ при движении по $C_{R}$ имеем $\Delta \operatorname{Arg} \varphi(p) \rightarrow \pi$ при $R \rightarrow \infty$, и, кроме того, $\varphi(0)>0$.

Рассмотрим движение по отрезку $p=-i \omega, 0 \leqslant \omega \leqslant R$. Обозначим

$$
\begin{aligned}
& u(\omega)=\operatorname{Re} \varphi(-i \omega)=\int_{0}^{\infty} a(\tau) \cos \omega \tau d \tau, \\
& v(\omega)=\operatorname{Im} \varphi(-i \omega)=-\omega+\int_{0}^{\infty} a(\tau) \sin \omega \tau d \tau .
\end{aligned}
$$

И здесь, как в доказательстве теоремы 1 , для завершения доказательства достаточно показать, что из условий $u(\omega)=0, \omega>0$ следует $v(\omega)<0$. Пусть $u(\omega)=0, \omega>0$. Умножим обе части неравенства

$$
\omega \tau+\cos \omega \tau-\left(\frac{\pi}{2}\right) \sin \omega \tau \geqslant 0, \quad \omega \tau \geqslant 0,
$$

на $a(\tau)$ и проинтегрируем по интервалу $[0, \infty)$. Получим

$$
\omega \int_{0}^{\infty} \tau a(\tau) d \tau+\int_{0}^{\infty} a(\tau) \cos \omega \tau d \tau-\left(\frac{\pi}{2}\right) \int_{0}^{\infty} a(\tau) \sin \omega \tau d \tau \geqslant 0
$$

Отсюда в силу (6), равенства $u(\omega)=0$ и условия $\int_{0}^{\infty} \tau a(\tau) d \tau<\pi / 2$ получим

$$
\left(\frac{\pi}{2}\right) \int_{0}^{\infty} a(\tau) \sin \omega \tau d \tau \leqslant \omega \int_{0}^{\infty} \tau a(\tau) d \tau<\frac{\omega \pi}{2},
$$

вследствие чего $v(\omega)<0$. Теорема 3 доказана.

Постоянная $\pi / 2$ в теореме 3 неулучшаема. Однако для любого $M>0$ можно подобрать такое ядро $a(\tau)$, что $\int_{0}^{\infty} \tau a(\tau) d \tau>M$, но нулевое решение уравнения (3) устойчиво. Поэтому никакое ограничение сверху величины $\int_{0}^{\infty} \tau a(\tau) d \tau$ не является необходимьм для устойчивости. 


\section{СПИСОК ЦИТИРОВАННОЙ ЛИТЕРАТУРЫ}

1. Gopalsamy K. Stability and Oscillations in Delay Differential Equations of Population Dynamics: Kluwer Academic Publishers, 1992. 2. Эльсгольц Л.Э., Норкин С. Б. Введение в теорию дифференциальных уравнений с отк лоняющимся аргументом. М.: Наука, 1964. 3. Мышкис А. Д. Линейные дифференциальные уравнения с запаздывающим аргументом. М.: Наука, 1972.

Челябинский государственный педагогический университет

Поступило

E-mail: mvagina@cspi.urc.ac.ru, kipnis@cspi.urc.ac.ru 